\title{
ON PROPERTIES OF ROSENTHAL COMPACTA
}

\author{
WITOLD MARCISZEWSKI
}

(Communicated by Dennis Burke)

\begin{abstract}
A compact space $K$ is a Rosenthal compactum if $K$ can be embedded in the space $B_{1}(P)$ of the first Baire class functions on $P$, the irrationals, endowed with the pointwise topology. We show that if $L$ is compact, $C_{p}(L)$ (the space of continuous real-valued functions on $L$ with the pointwise topology) is a continuous image of $C_{p}(K)$ and $K$ is a Rosenthal compactum, then $L$ is also. We prove that in some subclass of Rosenthal compacta (compacta consisting of the first Baire class functions with countable supports) the countable chain condition implies separability. We also show that compacta from this class possess a certain covering property hereditarily.
\end{abstract}

\section{INTRODUCTION}

In this paper we show ( $\S 3)$ that if $K$ is a Rosenthal compactum and $L$ is a compact space such that the function space $C_{p}(L)$ is a continuous image of the space $C_{p}(K)$, then $L$ is also a Rosenthal compactum (see $\S 2$ for the definitions). A similar result holds for these function spaces with the weak topology. This extends some results of Godefroy [Go] and the author [Ma3].

Next we consider a subclass of Rosenthal compacta consisting of the compacta that can be embedded in the intersection of the space $B_{1}(P)$ and the $\Sigma$-product $\Sigma(P)$ (this class contains all Gul'ko compacta of weight $\leq 2^{\omega}$ ). We prove $(\S 4)$ that for such compacta the property c.c.c. implies separability. This extends a result of Argyros and Negrepontis [AN]. We also show ( $\$ 5)$ that compacta from this class are hereditarily weakly $\theta$-refinable. This answers a question due to Gruenhage [Gr3].

\section{NOTATION AND TERMINOLOGY}

Our terminology follows Engelking [En ] and Negrepontis [Ne]. We denote by $R$ the real line, by $P$ the space of irrationals, by $Q$ the space of rationals, and by $\omega$ the set of natural numbers.

Given a set $\Gamma$, we denote by $R^{\Gamma}$ the Tychonoff product of the real line. The $\Sigma$-product of the real line is the subspace $\Sigma(\Gamma)$ of the product $R^{\Gamma}$ consisting of points with all but countably many coordinates equal to 0 , and let $c_{0}(\Gamma)=$ $\left\{x \in R^{\Gamma}\right.$ : for every $\varepsilon>0$ the set $\{\gamma:|x(\gamma)|>\varepsilon\}$ is finite $\} \subset \Sigma(\Gamma)$.

Received by the editors December 4, 1989.

1991 Mathematics Subject Classification. Primary 54C35; Secondary 54D20, 54D65.

Presented at the 23rd Annual Spring Topology Conference, March 16-18, 1989. 
For a compact space $K$ we denote by $C_{w}(K)$ the Banach space of continuous real-valued functions on $K$, endowed with the weak topology, and $C_{p}(K)$ is the space of continuous functions on $K$, equipped with the pointwise topology.

Let $X$ be a separable metrizable space. A function $f: X \rightarrow R$ is of the first Baire class iff $f^{-1}(U)$ is an $F_{\sigma}$-set for every open $U \subset R$ (cf. [Ku, $\left.\S 31\right]$ ). We denote by $B_{1}(X)$ the space of real-valued first Baire class functions on $X$ equipped with the topology of pointwise convergence. $B_{1}^{\omega}(X)$ is the intersection of the space $B_{1}(X)$ and $\Sigma(X)$.

A compact space $K$ is called a Rosenthal compactum if $K$ can be embedded in the space $B_{1}(P)$.

We will also consider the following classes of compact spaces: Corson (Eberlein) compacta, i.e., compact spaces that can be embedded in some $\Sigma(\Gamma)\left(c_{0}(\Gamma)\right)$. The class of Eberlein compacta coincides with the class of weakly compact subsets of Banach spaces (cf. [Ne]).

A compact space $K$ is $\mathrm{Gul}^{\prime}$ ko compact if the space $C_{p}(K)$ is countably determined, i.e., the continuous image of a closed subset of the product of some subset $S$ of the irrationals $P$ and a compact space (cf. [Ne]).

Each Eberlein compactum is $\mathrm{Gul}^{\prime} \mathrm{ko}$, and every Gul'ko compactum is Corson (see [Ne, Theorems 6.9, 6.10, and 6.23]). From a characterization of Gul'ko compacta due to Mercourakis [Me, Theorem 3.1], it follows that every Gul'ko compactum of the weight $\leq 2^{\omega}$ is Rosenthal (in fact, can be embedded in the space $\left.B_{1}^{\omega}(P)\right)$.

\section{Function SPACES ON Rosenthal compacta}

Using the ideal similar as in [Go, Proof of Proposition 7] we shall prove the following:

3.1. Theorem. Let $K, L$ be compact spaces such that the space $C_{p}(L)$ is a continuous image of the space $C_{p}(K)$. If $K$ is a Rosenthal compactum then so is $L$.

Proof. Without loss of generality we may assume that $K \subset B_{1}(P)$ and

$$
\exists x_{0} \in P \quad \forall k \in K \quad k\left(x_{0}\right)=1 .
$$

(If necessary we can take the second copy $P^{\prime}$ of the irrationals and extend all functions from $K$ over the discrete sum of $P$ and $P^{\prime}$ by putting value 1 on $\left.P^{\prime}\right)$.

Let $\varphi: C_{p}(K) \rightarrow C_{p}(L)$ be a continuous onto map.

For each finite sequence $x_{1}, \ldots, x_{n}$ of points of $P$ we define a continuous function $g_{x_{1}, \ldots, x_{n}}: K \rightarrow R$ by the formula

$$
g_{x_{1}, \ldots, x_{n}}(k)=k\left(x_{1}\right) \cdots k\left(x_{n}\right) \quad \text { for } k \in K \text {. }
$$

Let $A=\left\{g_{x_{1}, \ldots, x_{n}}: x_{1}, \ldots, x_{n} \in P, n=1,2, \ldots\right\}$. The functions from the set $A$ separate the points of $K$, and, by $(*), A$ contains the function identically equal to 1 on $K$. Hence from the Stone-Weierstrass theorem it follows that the set $B=\left\{\sum_{i=1}^{m} q_{i} g_{i}: q_{i} \in Q, g_{i} \in A, m=1,2, \ldots\right\}$ is dense in the Banach space $C(K)$, and consequently $B$ is dense in the space $C_{p}(K)$.

Let $T=\left\{\left(q_{1}, \ldots, q_{m}, n_{1}, \ldots, n_{m}\right): q_{i} \in Q, n_{i} \in \omega, n_{i} \geq 1\right.$, and $m=$ $1,2, \ldots\}$.

For every $\sigma \in T, \sigma=\left(q_{1}, \ldots, q_{m}, n_{1}, \ldots, n_{m}\right)$. let $Y_{\sigma}=P^{n_{1}} \times \cdots \times P^{n_{m}}$. 
Now, we define the space $Y$ as a discrete sum of spaces $Y_{\sigma}$, for $\sigma \in T$. Since the set $T$ is countable, the space $Y$ is homeomorphic to $P$.

We shall show that $L$ is a Rosenthal compactum by constructing an embedding of $L$ into $B_{1}(Y)$.

Let us define a map $h: L \rightarrow R^{Y}$ by the formula

$$
h(l)(y)=\varphi\left(\sum_{i=1}^{m} q_{i} g_{x_{1}^{i}, \ldots, x_{n_{i}}^{i}}\right)(l)
$$

for $l \in L, y \in Y_{\sigma}, \sigma \in T, \sigma=\left(q_{1}, \ldots, q_{m}, n_{1}, \ldots, n_{m}\right)$, and $y=$ $\left(\left(x_{1}^{1}, \ldots, x_{n_{1}}^{1}\right), \ldots,\left(x_{1}^{m}, \ldots, x_{n_{m}}^{m}\right)\right)$.

First, let us observe that $h$ is continuous because $\varphi\left(\sum_{i=1}^{m} q_{i} g_{x_{1}^{i}, \ldots, x_{n_{i}}^{i}}\right) \in$ $C_{p}(L)$. The fact that the set $\varphi(B)$ is dense in $C_{p}(L)$ implies that $h$ is one to one. Hence $h$ is a homeomorphic embedding, $L$ being compact.

It remains to show that for fixed $l \in L$ the function $h(l)(\cdot): Y \rightarrow R$ is of the first Baire class.

We shall consider a map $u: C_{p}(K) \rightarrow R$ defined by

$$
u(f)=\varphi(f)(l) \quad \text { for } f \in C_{p}(K) .
$$

Since $C_{p}(K)$ is a dense subset of the product $R^{K}$ by [Is, Corollary, p. 222], the map $u$ depends on countably many coordinates, i.e., there is a countable subset $S \subset K$ and a continuous map $v: \pi_{S}\left(C_{p}(K)\right) \rightarrow R$ such that $u=v \circ \pi_{S} \mid C_{p}(K)$, where $\pi_{S}$ denotes the projection of $R^{K}$ onto $R^{S}$. Let $S=\left\{k_{j}: j \in \omega\right\}$.

For every $k \in K$ we define a function $k^{\prime}: Y \rightarrow R$ by the formula

$$
\begin{gathered}
k^{\prime}(y)=\sum_{i=1}^{m} q_{i} g_{x_{1}^{i}, \ldots, x_{n_{i}}^{i}}(k)=\sum_{i=1}^{m} q_{i} k\left(x_{1}^{i}\right) \ldots . k\left(x_{n_{i}}^{i}\right) \quad \text { for } y \in Y_{\sigma}, \sigma \in T, \\
\sigma=\left(q_{1}, \ldots, q_{m}, n_{1}, \ldots, n_{m}\right) \text {, and } y=\left(\left(x_{1}^{1}, \ldots, x_{n_{1}}^{1}\right), \ldots,\left(x_{1}^{m}, \ldots, x_{n_{m}}^{m}\right)\right) .
\end{gathered}
$$

The functions $k^{\prime}$ are of the first Baire class. Hence, the diagonal map $\Delta_{j \in \omega} k_{j}^{\prime}: Y \rightarrow R^{s}$ defined by

$$
\Delta_{j \in \omega} k_{j}^{\prime}(y)\left(k_{j}\right)=k_{j}^{\prime}(y) \quad \text { for } y \in Y, k_{j} \in S
$$

is also of the first Baire class.

Finally, the equality

$$
h(l)(y)=v \circ \Delta_{j \in \omega} k_{j}^{\prime}(y) \quad \text { for } y \in Y
$$

shows that $h(l)(\cdot) \in B_{1}(Y)$.

3.2. Remark. The special case of Theorem 3.1, for separable Rosenthal compactum $K$ and homeomorphic function spaces $C_{p}(K)$ and $C_{p}(L)$ was proved in [Ma3, Theorem 3.5].

The assumption of compactness of the space $L$ in Theorem 3.1 is essential because the spaces $C_{p}([0,1])$ and $C_{p}(R)$ are homeomorphic, see [GKh, Theorem 4].

The results analogous to Theorem 3.1 are also true for Eberlein, Gul'ko, and Corson compacta (cf. [Ar, Corollaries 9 and 10]).

Theorem 3.1 will remain true if we replace the pointwise topology in the function spaces by the weak topology. 
3.3. Theorem. Let $K, L$ be compact spaces such that the space $C_{W}(L)$ is a continuous image of the space $C_{W}(K)$. If $K$ is a Rosenthal compactum then $L$ is also.

Proof. The proof for the case of pointwise topology requires only slight modification. First, without loss of generality, we may additionally assume that $K \subset B_{1}(P)$ is uniformly bounded. Let $B_{K}$ denote the unit ball of the space $M(K)=C(K)^{*}$ of Radon measures on $K$. We can consider $C_{W}(K)$ as a linear subspace of the product $R^{B_{K}}$.

Let $K^{\prime}=\left\{k^{\prime}: Y \rightarrow R: k \in K\right\}$ and $-K^{\prime}=\left\{-k^{\prime}: k^{\prime} \in K^{\prime}\right\}$ (we adopt the notation from the proof of Theorem 3.1). Because the set $B$ is dense in the Banach space $C(K)$, we can identify the space $B_{K}$, endowed with the weak* topology, with the closure of the convex hull of $K^{\prime} \cup-K^{\prime}$ in the space $B_{1}(Y)$ (cf. [Go, proof of Proposition 7]).

Now, using [Ma2, Lemma 4.1] we can factorize the map $u: C_{W}(K) \rightarrow R$ through the product $R^{s}$, for some countable set $S \subset B_{K}$. It remains to observe that for every $\mu \in S$ the map

$$
y \mapsto \mu(y)=\mu\left(\sum_{i=1}^{m} q_{i} g_{x_{1}^{i}, \ldots, x_{n_{i}}^{i}}\right)
$$

for $y \in Y_{\sigma}, \sigma \in T, \sigma=\left(q_{1}, \ldots, q_{m}, n_{1}, \ldots, n_{m}\right)$, and $y=\left(\left(x_{1}^{1}, \ldots, x_{n_{1}}^{1}\right)\right.$, $\left.\ldots,\left(x_{1}^{m}, \ldots, x_{n_{m}}^{m}\right)\right)$ is of the first Baire class.

3.4. Remark. Theorem 3.3 extends the results of Godefroy [Go, Proposition 10] and the author [Ma3, Theorem 3.1].

For the classes of Eberlein and $\mathrm{Gul}^{\prime}$ ko compacta, the results analogous to Theorem 3.3 are also true. Under the assumption of the Martin's Axiom (MA) and the negation of the continuum hypothesis $(\mathrm{CH})$, one can also prove similar result for Corson compacta, (cf. also [AMN2, Problem, p. 218]).

\section{SeParability and c.C.C.}

Let us recall that a topological space $X$ satisfies the countable chain condition (c.c.c) if every family of pairwise disjoint nonempty open subsets of $X$ is countable.

We shall discuss the following question raised by Pol [Po, §4, Remark 3.4] and Negrepontis [Ne, Problem 1.21]:

\section{Let $K$ be a c.c.c. Rosenthal compactum. Is $K$ separable?}

Since Rosental compacta are Fréchet topological spaces (cf. [BFT, Theorem $3 \mathrm{~F}]$ ), under $[\mathrm{Ma}+(\neg \mathrm{CH})]$ the answer to the above question is positive, see [CN, p. 201] and [Ju, Corollary, p. 76]. Godefroy proved that if a Rosenthal compactum $K$ has a strictly positive (Radon) measure $\mu$ (i.e., $\mu(U)>0$ for every nonempty open $U$ in $K$ ), then $K$ is separable (cf. [Go, Proposition 8]). Argyros and Negrepontis [AN] showed that c.c.c. Gul'ko compacta are separable. Analogous statement for the class of Corson compacta is independent of ZFC. Under [MA $+(\neg \mathrm{CH})]$ all c.c.c. Corson compacta are separable (cf. [CN, p. 205]). But, assuming $[\mathrm{CH}]$ there are many examples of nonseparable c.c.c. Corson compacta even Corson compacta with a strictly positive measure (cf. [AMN1, AMN2, CN, Ne]). 
We shall prove that for some subclass of Rosenthal compacta the answer to Negrepontis and Pol's question is positive.

4.1. Theorem. If $K$ is a compact c.c.c. subspace of the space $B_{1}^{\omega}(P)$ then $K$ is separable.

Proof. Assume, toward contradiction, that $K$ is nonseparable. Then there is an $\varepsilon>0$ and an uncountable subset $X \subset P$ such that for each $p \in X$ the set $V_{p}=\{k \in K:|k(p)|>\varepsilon\}$ is nonempty.

For every finite subset $A$ of $X$ we define an open set $V_{A}=\bigcap\left\{V_{p}: p \in A\right\}$. We will consider the space $X^{<\omega}$, of all nonempty finite subsets of $X$, equipped with the Vietoris topology, hence $X^{<\omega}$ is separable metrizable space (cf. [Ku, $\S 17,42)]$.

Let $\mathscr{A}=\left\{A \in X^{<\omega}: V_{A} \neq \varnothing\right\}$.

The following definition plays a crucial role in the proof:

We say that the set $A \in \mathscr{A}$ satisfies property (*) iff for every open neighborhood $U$ of $A$ in the space $X^{<\omega}$ there is an uncountable family $\mathscr{B} \subset \mathscr{A} \cap U$ such that

(i) $\forall B, C \in \mathscr{B} \quad(B \neq C \Rightarrow B \cap C=\varnothing)$ and

(ii) $\forall B \in \mathscr{B} \cup A \in \mathscr{A}$.

We need the following

Claim. There is a countable subset $Y \subset X$ such that for every $A \in \mathscr{A}$, disjoint from $Y, A$ satisfies $(*)$.

Suppose converse, then, by the transfinite induction we can construct an uncountable disjoint subfamily $\mathscr{B} \subset \mathscr{A}$ such that every $A \in \mathscr{B}$ does not satisfy $(*)$. Let $\mathscr{U}$ be a countable base for $X^{<\omega}$. For every $A \in \mathscr{B}$ there is a neighborhood $U_{A} \in \mathscr{U}$ of $A$ such that

$$
\text { the set }\left\{B \in \mathscr{B}: B \in U_{A} \text { and } B \cup A \in \mathscr{A}\right\} \text { is countable. }
$$

For some $U \in \mathscr{U}$ the family $\mathscr{C}=\left\{A \in \mathscr{B}: U_{A}=U\right\}$ is countable. Now, using (1) we can choose by transfinite induction an uncountable subfamily $\mathscr{D} \subset \mathscr{C}$ such that for every $A, B \in \mathscr{D}$ if $A \neq B$ then $A \cup B \notin \mathscr{A}$. Then the family $\left\{V_{A}: A \in \mathscr{D}\right\}$ is an uncountable family of nonempty pairwise disjoint open subset of $K$, which contradicts the c.c.c. property of $K$.

Using the claim one can easily construct, by induction, a sequence $\left(A_{n}\right)_{n \in \omega}$, $A_{n} \in \mathscr{A}$, satisfying the following conditions for every $n \in \omega$ :

(a) $A_{n} \cap Y=\varnothing$,

(b) $A_{n} \subset A_{n+1}$ (hence $V_{A_{n}} \supset V_{A_{n+1}}$ ),

(c) $A_{n}$ is $n^{-1}$-dense in itself, i.e., $\forall p \in A_{n} \exists q \in A_{n} \quad(p \neq q$ and $\operatorname{dist}(p, q)$ $\left.<n^{-1}\right)$.

Then the set $S=\bigcup\left\{A_{n}: n \in \omega\right\}$ is dense in itself, and $\bigcap\left\{\operatorname{cl} V_{A_{n}}: n \in \omega\right\} \neq \varnothing$. If $k \in \bigcap\left\{\mathrm{cl} V_{A_{n}}: n \in \omega\right\}$ then $|k(p)| \geq \varepsilon$ for all $p \in S$. But for every $k \in B_{1}^{\omega}(P)$ the set $\{p \in P:|k(p)| \geq \varepsilon\}$ is a countable $G_{\delta}$-set in $P$, hence scattered-a contradiction.

4.2. Remark. Theorem 4.1 extends the result of Argyros and Negrepontis concerning Gul'ko Compacta [AN] since each Gul'ko compactum of weight $\leq 2^{\omega}$ can be embedded in $B_{1}^{\omega}(P)$ (see $\S 2$ ), and c.c.c. compacta of countable tight have weight $\leq 2^{\omega}$ (cf. [Ho, Theorems 6.2 and 7.13]). However Gul'ko compacta possess a stronger property: Gruenhage [Gr3] and Leiderman [Le] proved 
that Gul'ko compacta have dense $G_{\delta}$ metrizable subsets. This result cannot be generalized for Corson compacta, Todercevic [To, Theorem 9.14] constructed (in ZFC) an example of a Corson compactum with no dense metrizable subspace. So it is natural to ask whether Rosenthal compacta have dense metrizable subspaces. Let us notice that Bourgain [Bo] showed that each Rosenthal compactum has a dense subspace consisting of points with countable base of neighborhoods.

\section{COVERING PROPERTY}

In this section we shall show the following:

5.1. Theorem. Every compact subset $K$ of the space $B_{1}^{\omega}(P)$ is hereditarily weakly $\theta$-refinable.

First, let us recall some definitions and make few comments. A topological space $X$ is weakly $\theta$-refinable if every open cover of $X$ has an open refinement $\mathscr{U}=\bigcup\left\{\mathscr{U}_{n}: n \in \omega\right\}$ such that for every $x \in X$ there is an $n \in \omega$ such that $0<\operatorname{ord}\left(x, \mathscr{U}_{n}\right)<\omega$. The space $X$ is meta-Lindelöf ( $\sigma$-metacompact) if every open cover of $X$ has a point-countable $(\sigma$-point-finite) open refinement (cf. [Bu]).

Yakovlev [Ya] proved that Corson compacta are hereditarily meta-Lindelöf and Eberlein compacta are hereditarily $\sigma$-metacompact. Gruenhage [Gr1] showed that for compact spaces $K$, the property that $K^{2}$ is hereditarily metaLindelöf $(\sigma$-metacompact) characterizes Corson (Eberlein) compacta. Gruenhage [Gr3] also proved that $\mathrm{Gul}^{\prime}$ ko compact spaces are hereditarily weakly $\theta$ refinable and gave an example of a Corson compactum without this property (cf. [Gr2]). In [Gr3] he asked a question if the property that $K^{2}$ is hereditarily weakly $\theta$-refinable characterizes $\mathrm{Gul}^{\prime} \mathrm{ko}$ compacta within the class of Corson compacta $K$. Theorem 5.1 gives a negative answer to this question, since there are examples of compact subsets of $B_{1}^{\omega}(P)$ that are not Gul'ko compacta (see [AP, Ma1, AMN2, Me, Le]). We do not know if all Rosenthal compacta are hereditarily weakly $\theta$-refinale. Gardner [Ga] showed that every compact hereditarily weakly $\theta$-refinable space $K$ with no discrete subspace of measurable cardinality is Radon, i.e., every Borel measure on $K$ is regular. Hence Theorem 5.1 has the following

5.2. Corollary. Compact subspaces of the space $B_{1}^{\omega}(P)$ that do not contain discrete subsets of measurable cardinality are Radon.

Before we start the proof of Theorem 5.1 we shall show the following technical lemma.

5.3. Lemma. Let $K \subset \Sigma(\Gamma)$ be a Corson compactum such that $0 \in K$ and $\mathscr{V}$ be a family of basic neighborhoods of 0 in $K$, i.e., for every $V \in \mathscr{V}$ there is a finite sequence $\gamma_{1}^{v}, \ldots, \gamma_{n_{v}}^{v} \in \Gamma$ and an $\varepsilon_{v}>0$ such that $V=\left\{f \in K:\left|f\left(\gamma_{i}^{v}\right)\right|<\right.$ $\varepsilon_{v}$ for $\left.i=1, \ldots, n_{v}\right\}$. Then there exists a countable subfamily $\mathscr{W} \subset \mathscr{V}$ such that $\bigcup \mathscr{W}=\bigcup \mathscr{V}$.

Proof. Without loss of generality we may assume that there is an $n \geq 1$ and $\varepsilon>0$ such that $n_{v}=n$ and $\varepsilon_{v}=\varepsilon$ for all $V \in \mathscr{V}$.

We can prove the existence of subfamily $\mathscr{W}$ by induction on $n$. For $n=$ 
1 , if $|\mathscr{V}|>\omega$ then $\mathscr{V}$ covers $K$ since $K \subset \Sigma(\Gamma)$. Hence we can apply compactness of $K$.

Now let us assume that the lemma holds for $n-1$ where $n>1$. We shall consider two cases:

$1^{\circ}$. There is an uncountable subfamily $\mathscr{V}^{0} \subset \mathscr{V}$ such that for distinct $V, W \in \mathscr{V}$, the sets $\left\{\gamma_{1}^{v}, \ldots, \gamma_{n}^{v}\right\}$ and $\left\{\gamma_{1}^{w}, \ldots, \gamma_{1}^{w}\right\}$ are disjoint. Then $K=\bigcup \mathscr{V}^{0}$ and we can again use compactness of $K$.

$2^{\circ}$. There is a countable set $A \subset \Gamma$ such that for all $V \in \mathscr{V},\left\{\gamma_{1}^{v}, \ldots, \gamma_{n}^{v}\right\} \cap$ $A \neq \varnothing$. Let $A=\left\{\gamma_{0}, \gamma_{1}, \ldots\right\}$ and $\mathscr{V}_{i}=\left\{V \in \mathscr{V}: \gamma_{i} \in\left\{\gamma_{1}^{v}, \ldots, \gamma_{n}^{v}\right\}\right\}$.

For every $i, k \in \omega, k>\varepsilon^{-1}$, we define a Corson compactum

$$
L_{i, k}=\left\{f\left|\Gamma-\left\{\gamma_{i}\right\}:\right| f\left(\gamma_{i}\right) \mid \leq \varepsilon-k^{-1}\right\} \subset \Sigma\left(\Gamma-\left\{\gamma_{i}\right\}\right) .
$$

Next, for each $V \in \mathscr{V}_{i}$ we take $V_{i, k}=\left\{f \in L_{i, k}:|f(\gamma)|<\varepsilon\right.$ for $\gamma \in$ $\left.\left\{\gamma_{1}^{v}, \ldots, \gamma_{n}^{v}\right\} \backslash\left\{\gamma_{i}\right\}\right\}$.

Now we can apply the inductive hypothesis for compactum $L_{i, k}$ and the family $\mathscr{V}_{i, k}=\left\{V_{i, k}: V \in \mathscr{V}_{i}\right\}$ and obtain a countable subfamily $\mathscr{W}_{i, k} \subset \mathscr{V}_{i, k}$ such that $\bigcup \mathscr{W}_{i, k}=\bigcup \mathscr{V}_{i, k}$.

Finally, as a countable subcover of $\bigcup \mathscr{V}$ we can take $\left\{V: V_{i, k} \in \mathscr{W}_{i, k}, i\right.$, $\left.k \in \omega, k>\varepsilon^{-1}\right\}$.

Proof of Theorem 5.1. Let $K \subset B_{1}^{\omega}(P)$ be compact. It is enough to show that any open subset $A$ of $K$ is weakly $\theta$-refinable. Let $\mathscr{U}$ be an open cover of $A$.

We choose a base $\left(W_{n}\right)_{n \in \omega}$ of the space $R-\{0\}$ such that $0 \notin \mathrm{cl} W_{n}$ for all $n \in \omega$. We may assume that for every $U \in \mathscr{U}$ there exist $p_{1}, \ldots, p_{k}$, $q_{1}, \ldots, q_{l} \in P, m, m_{1}, \ldots, m_{k} \in \omega, m \geq 1$ such that

$$
\begin{aligned}
& U=\left\{f \in K: \forall j \leq l,\left|f\left(q_{j}\right)\right|<m^{-1}\right\} \quad \text { or } \\
& U=\left\{f \in K: \forall i \leq k, f\left(p_{i}\right) \in W_{m_{i}} \text { and } \forall j \leq l,\left|f\left(q_{j}\right)\right|<m^{-1}\right\} .
\end{aligned}
$$

First we shall simplify the form of sets $U$. For every $m_{1}, \ldots, m_{k} \in \omega$ let $Z=\left\{\left(x_{1}, \ldots, x_{k}\right) \in R^{k}: \exists i \leq k, x_{i}=0\right\}$ and let $\varphi_{m_{1}}, \ldots, m_{k}: R^{k} \rightarrow[0, \infty)$ be a continuous function such that $\varphi_{m_{1}, \ldots, m_{k}}(Z)=\{0\}$ and $\left.\varphi_{m_{1}}^{-1} \ldots, m_{k}(1, \infty)\right)=$ $W_{m_{1}} \times \cdots \times W_{m_{k}}$. Now let $S$ be a discrete sum of $P$ and all products $P_{m_{1}} \times$ $\cdots \times P_{m_{k}}$ for $m_{1}, \ldots, m_{k} \in \omega$, where each space $P_{m_{i}}$ is a topological copy of the irrationals $P$. The space $S$ is homeomorphic to $P$.

For every $f \in K$ we define a map $f^{\prime}: S \rightarrow R$ in such a way that $f^{\prime} \mid P \equiv f$ and $f^{\prime} \mid P_{M_{1}} \times \cdots \times P_{m_{k}} \equiv \varphi_{m_{1}, \ldots, m_{k}} \circ(f \times \cdots \times f)$.

One can easily check that the space $L=\left\{f^{\prime}: f \in K\right\}$ is a subspace of $B_{1}^{\omega}(S)$, which can be identified with $K$.

For $U \in \mathscr{U}$, let $U^{\prime}=\left\{f^{\prime}: f \in U\right\}$ and $\mathscr{U}^{\prime}=\left\{U^{\prime}: U \in \mathscr{U}\right\}$. Now, for every $U^{\prime} \in \mathscr{U}^{\prime}$ there exist $s^{u}, q_{1}^{u}, \ldots, q_{l}^{u} \in S$ and $m \in \omega, m \geq 1$ such that

$$
\begin{aligned}
& U^{\prime}=\left\{g \in L: \forall j \leq l,\left|f\left(g_{j}^{u}\right)\right|<m^{-1}\right\} \quad \text { or } \\
& U^{\prime}=\left\{g \in L: g\left(s^{u}\right)>1 \text { and } \forall j \leq l,\left|f\left(q_{j}^{u}\right)\right|<m^{-1}\right\} .
\end{aligned}
$$

Let $\mathscr{U}_{0}^{\prime}$ be the family of sets $U^{\prime}$ of the first form and $\mathscr{U}_{1}^{\prime}$ consists of $U^{\prime \prime}$ s of the second form.

By Lemma 5.3 there is a countable subfamily $\mathscr{U}_{0}^{\prime \prime}=\left\{U_{0, i}^{\prime}: i \in \omega\right\} \subset \mathscr{U}_{0}^{\prime}$ with $\cup \mathscr{U}_{0}^{\prime \prime}=\bigcup \mathscr{U}_{0}^{\prime}$.

For each $s \in\left\{s^{u}: U \in \mathscr{U}_{1}^{\prime}\right\}$, let $\mathscr{U}_{s}^{\prime}=\left\{U^{\prime} \in \mathscr{U}_{1}^{\prime}: s^{u}=s\right\}$. Applying Lemma 5.3 for the sets $L_{s, k}=\left\{g \mid S-\{s\}: g \in L, g(s) \geq 1+k^{-1}\right\}$ for $k=1,2, \ldots$, 
we can find a countable subfamily $\mathscr{U}_{s}^{\prime \prime} \subset \mathscr{U}_{s}^{\prime}$ such that $\bigcup \mathscr{U}_{s}^{\prime \prime}=\bigcup \mathscr{U}_{s}^{\prime}$. Let $\mathscr{U}_{s}^{\prime \prime}=\left\{U_{s, 0}^{\prime}, U_{s, 1}^{\prime}, \ldots\right\}$ and let $\left(Y_{n}\right)_{n \in \omega}$ be a base of $S$.

We shall define the weak $\theta$-refinement $\mathscr{V}$ of $\mathscr{U}^{\prime}$ in the following way:

$$
\mathscr{V}_{n, i}=\left\{U_{s, i}^{\prime}: s \in Y_{n}\right\} \cup\left\{U_{0, i}^{\prime}\right\} \quad \text { and } \quad \mathscr{V}=\bigcup\left\{\mathscr{V}_{n, i}: n, i \in \omega\right\}
$$

Let $g \in \bigcup U_{1}^{\prime}$. The set $T=\{s \in S: g(s) \geq 1\}$ is a countable $G_{\delta}$ in $S$, hence scattered.

For $\alpha>\omega_{1}$ let us denote by $T^{(\alpha)}$ the $\alpha$ th derived set of $T$, see [Ku, $\left.\S 24 . I V\right]$. If $s \in T$ let $\alpha_{s}<\omega_{1}$ be such that $s \in T^{\left(\alpha_{s}\right)} \backslash T^{\left(\alpha_{s}+1\right)}$. We choose $s_{0} \in T$ such that there is an $i_{0} \in \omega$ with $g \in U_{s_{0}, i_{0}}^{\prime}$ and $\alpha_{s_{0}}$ is minimal. Suppose that for all $n \in \omega$ such that $s_{0} \in Y_{n}, \operatorname{ord}\left(g, \mathscr{V}_{n, i_{0}}\right) \geq \omega$. Then for all $n$ with $s \in Y_{n}$ the set $\left\{s \in T \cap Y_{n}: g \in U_{s, i_{0}}^{\prime}\right\}$ is infinite, which contradicts the minimality of $\alpha_{s_{0}}$.

\section{REFERENCES}

[AP] K. Alster and R. Pol, On function spaces of compact subsets of $\Sigma$-products of the real line, Fund. Math. 107 (1980), 135-143.

[Ar] A. V. Arhangel'skii, Some results and problems in $C_{p}(X)$-theory, Proc. Sixth Prague Topol. Sympos. 1986, Heldermann Verlag, Berlin, 1988, pp. 11-31.

[AN] S. Argyros and S. Negrepontis, On weakly $K$-countable determined spaces of continuous functions, Proc. Amer. Math. Soc. 87 (1983), 731-736.

[AMN1] S. Argyros, S. Mercourakis, and S. Negrepontis, Analytic properties of Corson-compact spaces, Proc. Fifth Prague Topol. Sympos. 1981, Heldermann Verlag, Berlin, 1982, pp. $12-23$.

[AMN2] _ Functional-analytic properties of Corson-compact spaces, Studia Math. 89 (1988), 197-229.

[Bo] J. Bourgain, Some remarks on compact sets of the first Baire class, Bull. Soc. Math. Belg. 30 (1978), 3-10.

[BFT] J. Bourgain, D. H. Fremlin, and M. Talagrand, Pointwise compact sets of Baire-measurable functions, Amer. J. Math. 100 (1978), 845-886.

[Bu] D. Burke, Covering properties, Handbook of Set-Theoretic Topology, North-Holland, Amsterdam, 1984, pp. 347-422.

[CN] W. W. Comfort and S. Negrepontis, Chain conditions in topology, Cambridge Tracts in Math., vol. 79, Cambridge Univ. Press, Cambridge, UK, 1982.

[En] R. Engelking, General topology, PWN, Warsaw, 1977.

[Ga] R. J. Gardner, The regularity of Borel measures and Borel measure compactness, Proc. London Math. Soc. (3) 30 (1975), 95-113.

[Go] G. Godefroy, Compacts de Rosenthal, Pacific J. Math. 91 (1980), 293-306.

[Gr1] G. Gruenhage, Covering properties on $X^{2}-\Delta, W$-sets, and compact subsets of $\Sigma$-products, Topology Appl. 17 (1984), 287-304.

[Gr2] _-, On a Corson compact space of Todercevic, Fund. Math. 126 (1986), 261-268.

[Gr3] _-, A note on Gul'ko compact spaces, Proc. Amer. Math. Soc. 100 (1987), 371-376.

[GKh] S. P. Gul'ko and T. E. Khmyleva, Compactness if not preserved by t-equivalence, Mat. Z. 39 (1986), 895-903. (Russian)

[Ho] R. Hodel, Cardinal functions, Handbook of Set-Theoretic Topology, North-Holland, Amsterdam, 1984, pp. 1-62.

[Is] J. R. Isbell, Mazur's theorem, Proceedings of the Symposium held in Prague in September 1961, Publishing House of Czechoslovak Academy of Sciences, Prague, 1962, pp. 221-225.

[Ju] I. Juhasz, Cardinal functions in topology-ten years later, Math. Centre Tracts, vol. 123 Amsterdam, 1980. 
[Ku] K. Kuratowski, Topology vols. I and II, Academic Press : New York and London and PWN:Warsaw, 1966 and 1968.

[Le] A. G. Leiderman, Everywhere dense metrizable subspace of Corson compacta, Mat. Z. 38 (1985), 440-449.

[Ma1] W. Marciszewski, Lindelöf property in function spaces and a related selection theorem, Proc. Amer. Math. Soc. 101 (1987), 545-550.

[Ma2] _ A function $C(K)$ not weakly homeomorphic to $C(K) \times C(K)$, Studia Math. 88, (1988), 129-137.

[Ma3] _ On a classification of pointwise compact sets of the first Baire class functions, Fund Math. 133 (1989), 195-209.

[Me] S. Mercourakis, On weakly countably determined Banach spaces, Trans. Amer. Math. Soc. 300 (1987), 307-327.

[Ne] S. Negrepontis, Banach spaces and topology, Handbook of Set-Theoretic Topology, NorthHolland, Amsterdam, 1987, pp. 1045-1142.

[Po] R. Pol, On pointwise and weak topology in function spaces, University of Warsaw, No. 4/84, Warsaw 1984 preprint.

[To] S. Todorcevic, Trees and linearly ordered sets, Handbook of Set-Theoretic Topology, NorthHolland, Amsterdam, 1984, pp. 235-293.

[Ya] N. N. Yakovlev, On bicompacta in $\Sigma$-products and related spaces, Comment. Math. Univ. Carolin. 21 (1980), 263-282.

Department of Mathematics, University of Kansas, Lawrence, Kansas 66045

Current address: Department of Mathematics, University of Warsaw, Banacha 2, 02-097 Warsazawa, Poland

E-mail address: WMARCISZ@PLEARN.BITNET 